\title{
Neural stem cells reversed disease symptoms in an experimental autoimmune encephalomyelitis mouse model of multiple sclerosis
}

\section{Christina Brown}

Oakland University

Christina McKee

Oakland University

\section{Sophia Halassy}

Ascension Providence Hospital Novi Campus

\section{Suleiman Kojan}

Oakland University William Beaumont School of Medicine

\section{Douglas Feinstein}

University of Illinois at Chicago College of Medicine

\section{G. Rasul Chaudhry ( $\square$ chaudhry@oakland.edu )}

University of Illinois at Chicago College of Medicine https://orcid.org/0000-0003-1692-8420

\section{Research}

Keywords: Multiple sclerosis, neural stem cells, mesenchymal stem cell, experimental autoimmune encephalomyelitis, anti-inflammatory, remyelination, and neuroprotection

Posted Date: March 3rd, 2021

DOl: https://doi.org/10.21203/rs.3.rs-278712/v1

License: (c) (1) This work is licensed under a Creative Commons Attribution 4.0 International License. Read Full License 


\section{Abstract \\ Background}

Multiple sclerosis (MS) is an autoimmune inflammatory disease of the central nervous system (CNS). MS affects millions of people and causes a great economic and societal burden. Currently used treatment drugs have side effects and only address the symptoms but not the causes of MS. In this study, a novel approach of transplanting neural stem cells (NSCs) derived from human primitive mesenchymal stem cells (MSCs) was investigated in an experimental autoimmune encephalomyelitis (EAE) mouse model of MS.

\section{Methods}

Primitive MSCs were differentiated into NSCs using selective media. The cells were labeled with PKH26 and injected into the tail vein of EAE mice. The animals were evaluated for changes in neurobehavior and weight twice daily. Two weeks following cell transplantation, the animals were sacrificed to collect the blood, lymphatic and CNS tissues for analysis. FACS analysis was used to track labeled cells and infiltrates. Histochemical analysis was performed to determine the levels of myelination. Expression of inflammation, neural, astrogliosis, neuroprotection, and myelination markers was investigated by using immunohistochemical and qRT-PCR analyses.

\section{Results}

Neurobehavioral assays showed that EAE disease process was halted by transplantation of both MSCs and NSCs. However, NSCs showed greater efficacy in reversing the disease symptoms, which resulted in near complete recovery of EAE animals. Post-transplantation analyses also showed homing of transplanted cells into the CNS with concomitant induction of anti-inflammatory response resulting in reduction of immune infiltrates. Luxol fast blue staining intensity of CNS tissues was significantly improved in treated mice as compared to EAE animals, suggesting endogenous remyelination. NSC transplantation also modulated Treg and Th17 cells in EAE mice to levels comparable to healthy controls. In addition, several of the markers associated with neuroprotection (i.e. Igf, Bdnf, and Trkb), myelination (i.e. Erk2, Krox-20, Oct-6, Mpz, Mbp, and Mog) and neurogenesis (i.e. Tuj1 and Nestin) were upregulated, suggesting endogenous regeneration in treated animals.

\section{Conclusions}

Cell transplantation was more effective at an earlier point of EAE disease (EAE stage 1) than later (EAE stage 2). These promising results provide basis for large-scale clinical studies to treat MS using NSCs derived from primitive MSCs. 


\section{Introduction}

Multiple sclerosis (MS) is a chronic autoimmune inflammatory disease of the central nervous system (CNS) (1). In MS, inflammatory responses are elicited by the intrinsic immune system as well as by parenchymal glial cells, which contribute to oligodendrocyte damage and demyelination, axonal damage and impaired or reduced neuronal signaling (2). MS affects approximately 400,000 individuals in the United States and 2.5 million people worldwide and inflicts substantial economic and societal burden due to the early age of disease onset and recurrent relapses (3). Multiple factors including genetics and environmental agents seem to be involved with the onset of MS (2). Although, drug treatments help in reducing disease progression or minimizing disability in patients, they cause severe side effects and do not reverse the symptoms of MS (4). MS drugs pose serious side effects and only help in reducing severity of symptoms or slowing progression rather than treating the cause of disease (5). Therefore, it is imperative to design therapeutic strategies that not only stop disease progression, but also repair the damage to the CNS without severe side effects to MS patients. With the advent of recent progress in stem cell research, cell therapy could provide a promising alternative to drug therapy (6). Mesenchymal stem cells (MSCs) have shown paracrine effects by secreting cytokines and trophic factors involved in immunomodulation (7). MSCs are known to home towards the site of injury and participate in healing and repair processes throughout life (8). Therefore, there is tremendous interest in MSCs due to their medicinal properties. However, MSCs isolated from adult sources have poor growth, limited self-renewal and differentiation potential (8). Moreover, MSCs isolated from adult sources have poor growth, limited self-renewal and differentiation potential as well as pose significant risk of graft verse host disease (GvHD). On the other hand, MSCs isolated from perinatal sources are more naïve, have a low risk of host immune response due to the lack of expression of the major histocompatibility complex (MHC) class II antigens and thus do not cause GvHD (8). Recently, we have isolated highly proliferative primitive MSCs, which can be rapidly amplified and differentiated into functional derivatives for large-scale clinical studies (8-10). Although MSCs are known to home towards the site of injury or damage as well as secrete cytokines and growth factors that may indirectly promote endogenous tissue regeneration, transplanted MSCs and their derivatives crossing the blood brain barrier (BBB) to repair the damaged CNS is not well established (11). We sought to determine whether CNS damage due to MS could be repaired by augmentation of neural stem cells (NSCs). Several reports described the ability of NSCs migrate to the area of pathology in experimental models of CNS disease (12-14). Therefore, we applied a novel approach whereby primitive MSCs were differentiated into NSCs to investigate their effect on the disease process in treating an experimental autoimmune encephalitis (EAE) mouse model, which is known to share similar characteristics pathological features with MS (15). Our results demonstrate reversal of disease symptoms and significant improvement in the pathology of EAE animals transplanted with NSCs. This is the first report examining the anti-inflammatory responses, neuroprotection and endogenous regeneration of perinatal MSC derived NSCs.

\section{Materials And Methods}




\section{Maintenance and culture of primitive MSCs}

Previously isolated and well characterized primitive MSCs (10), were maintained using growth medium (GM) containing DMEM nutrient mix F12 medium (DMEM/F12; Life Technologies, Carlsbad, CA, USA), supplemented with $10 \%$ fetal bovine serum (FBS; VWR, Radnor, PA, USA), and $5.6 \%$ of antibiotic solution ( $0.1 \%$ gentamicin, $0.2 \%$ streptomycin, and $0.12 \%$ penicillin); (Sigma, St Louis, MO, USA) and incubated at $37^{\circ} \mathrm{C}$ in an atmosphere of $5 \% \mathrm{CO}_{2}$ in a humidified incubator. Cells were routinely analyzed using fluorescence-activated cell sorting (FACS) as previously described in our published results (10).

\section{Differentiation of primitive MSCs into NSCs}

Primitive MSCs were induced towards the neural lineage by culturing the cells in induction media containing $10 \mu \mathrm{g}$ epidermal growth factor (EGF; PeproTech, Rocky Hill, NJ, USA) in DMEM/F12 basal medium supplemented with $5.6 \%$ antibiotic solution for 3 days. Then replaced with neural media containing $20 \mu \mathrm{g}$ EGF, $20 \mathrm{ng}$ basic fibroblast growth factor (bFGF), $2 \mathrm{mM}$ Glutamine (Sigma), 1x B27 supplement (Thermo Fisher Scientific, Waltham, MA, USA), in neurobasal medium for 2 weeks. Characterization of NSCs for the expression of neural markers was performed by immunocytochemical staining, FACS, and quantitative reverse transcriptase polymerase chain reaction (qRT-PCR) analysis.

Immunocytochemical analysis: Cells were fixed with 4\% paraformaldehyde for 10 minutes at room temperature, permeabilized with $0.5 \%$ Triton X-100 (Sigma) and blocked with $2 \%$ bovine serum albumin (Sigma) for 1 hour. Cells were then stained with primary antibodies at 1:100 dilution: NESTIN, TUJ1, VIMENTIN, and PAX 6 at $4^{\circ} \mathrm{C}$ overnight, followed by staining of secondary antibodies at 1:200 dilution at room temperature for 2 hours. Cells were counterstained with DAPI at 1:100 dilution for 5 minutes at room temperature. Fluorescent images were captured using a confocal microscope (NIKON Instruments Inc, Melville, NY, USA). Fluorescent intensity and percentage of positive cells were calculated using ImageJ software (NIH, Bethesda, MD, USA).

Western blot analysis of cells: MSCs and NSCs were lysed for protein using RIPA buffer (Sigma) and quantified using the Pierce $660 \mathrm{~nm}$ protein assay on the NanoDrop 1000 spectrophotometer (Fisher Scientific). The lysate (30 $\mu \mathrm{g}$ of total proteins) was resolved using SDS-PAGE with $12 \%$ resolving gel and $6 \%$ stacking gel and transferred to a nitrocellulose membrane (BioRad) at a continuous current of $100 \mathrm{~V}$ for 90 minutes. For antibody staining, the membrane was blocked with $5 \%$ nonfat dry milk dissolved in TBS $1 \mathrm{X}$ containing $0.1 \%$ Tween-20 for 30 minutes and incubated with primary antibodies at a 1:500 dilution in the blocking solution overnight at $4^{\circ} \mathrm{C}$. The membrane was then washed with TBST and incubated with the secondary antibody conjugated with HRP at a 1:10000 dilution in the blocking solution for 2 hours at room temperature. After washing with TBST, the blot was stained with BioRad chemiluminescence for 5 minutes and bands were visualized using a chemidoc (BioRad). Band intensities were quantified using ImageJ software (NIH, Bethesda, MD, USA) and normalized to GAPDH.

Experimental animals and EAE induction: All animal experiments were approved by the Institutional Animal Care and Use Committee (IACUC), Oakland University, Rochester, Michigan (IACUC \#18081) and 
the Institutional Biosafety Committee (IBC), Oakland University, Rochester, Michigan (IBC \#2858). A total of $25 \mathrm{C} 57 \mathrm{BL} / 6 \mathrm{~J}$ mice from Jackson Laboratory were used for this study. EAE was induced in 6 weeks old $\mathrm{C} 57 \mathrm{BI} / 6$ mice by subcutaneous immunization with $200 \mu \mathrm{g}$ of $\mathrm{MOG}_{33-35}$ peptide in complete Freud's adjuvant containing 2-5 mg killed mycobacterium tuberculosis $\mathrm{H} 37 \mathrm{Ra} / \mathrm{mL}$ emulsion (Hooke Laboratories, Lawrence, MA, USA). Mice were also injected intraperitoneally (i.p.) with pertussis toxin (100 $\mathrm{ng}$, Hooke Laboratories) at the day of immunization and after 24 hours following the published protocol (16). Clinical score was blindly registered according to the following scale: 0: no clinical signs, 0.5: partial limp tail, 1: limp tail, 1.5: mild impaired righting, 2: severe impaired righting, 2.5: mild paresis of one hind limb, 3: severe paresis of one hind limb, 3.5: severe paresis of one hind limb and mild paresis of second hind limb, 4: paresis of two hind limbs, 4.5: paresis of both hind limb and partial fore limb, and 5: severe paralysis or death. Non-injected C57BL/6J mice were used as controls. All mice were maintained at the Oakland University Animal Facility under a 12/12-hour light and dark cycle.

\section{Cell transplantation in an EAE mouse model}

Cells were labeled with cell membrane labeling dye PKH26 (Sigma) following manufacturer's instructions (17). Confocal microscopy and FACS analysis were used to confirm the efficiency of the fluorescently labeled cells prior to transplantation. There were eight treatments $(n=6$ each), 1 . Untreated healthy, 2 , healthy + MSCs, 3. healthy + NSCs, 4. Untreated EAE, 5. EAE stage $1+$ MSCs, 6. EAE stage $1+$ NSCs, 7. EAE stage $2+$ MSCs, and 8 . EAE stage $2+$ NSCs. The experiments were performed in triplicates. The mice were restrained in a rodent restrainer for cell transplantation. $10^{6}$ primitive MSCs or NSCs were injected intravenously via the tail vein at either clinical scores of 1 or 2 . The mice were monitored for 15 days following cell transplantation. Mice were euthanized humanly and CNS, lymphoid tissues (spleen, liver, brain, and spinal cord), and blood were extracted.

\section{Tracking of transplanted cells}

Whole tissue and blood samples was place in red blood cell lysis buffer for 5 minutes and then homogenized in a centrifuge tube. Culturing medium was then added to the tube, inverted, and centrifuged at $1300 \mathrm{rpm}$ for 5 minutes at $4^{\circ} \mathrm{C}$. The supernatant was discarded, and cells were resuspended in PBS 1x. The cells were then filtered through a cell strainer. Labeled cells in each sample were determined using a FACS Canto II (Becton Dickinson) and Diva Software (Becton Dickinson).

Histological analysis: For paraffin embedding, brain and spinal cord tissue samples were fixed with $4 \%$ paraformaldehyde for 24 hours. They were then dehydrated in $70 \%$ ethanol for 1 hour, $95 \%$ ethanol for 1 hour, $100 \%$ ethanol for 4 hours, xylene for 2 hours, and paraffin for 3 days. Tissue samples were then imbedded in paraffin and sectioned (5-10 $\mu \mathrm{m}$ thick) using a microtome. The paraffin embedded samples were stained with hematoxylin and eosin (H\&E; Thermo Fisher Scientific) to evaluate the cellular structure of the tissue and Luxol fast blue (LFB; Sigma) to examine myelination. To detect the protein levels of the human neural and inflammatory markers expressed by mouse brain and spinal cord tissue, the sections were stained with primary antibodies 1:100 dilution: TUJ1, NESTIN, MOG, MBP, GFAP, CD45, CD68, and 
$\mathrm{CD} 3 \mathrm{E}$, respectively, at $4^{\circ} \mathrm{C}$ overnight followed by staining of secondary antibodies at 1:200 dilution at room temperature for 2 hours. They were counterstained with DAPI at 1:100 dilution for 5 minutes at room temperature. Fluorescent images of the stained sections were captured using a confocal microscope (NIKON Instruments Inc.).

\section{Quantification of Treg and Th17 cells in the blood, spleen, and CNS tissues}

For FACS analysis, whole tissue and blood samples were placed in red blood cell lysis buffer for 5 minutes and then homogenized. Culture medium was then added to the tube, inverted, and centrifuged at $1300 \mathrm{rpm}$ for 5 minutes at $4^{\circ} \mathrm{C}$. The supernatant was discarded, and cells were resuspended in PBS $1 \mathrm{X}$. The cells were then filtered through a cell strainer. Cells $\left(10^{6}\right)$ were stained with CD25, IL-17A (FITC labeled antibodies), or CD4 (APC labeled antibodies) (Becton Dickinson). Labeled cells were analyzed on a FACS Canto II (Becton Dickinson) using Diva Software (Becton Dickinson).

qRT-PCR analysis of cells and mouse tissue: Isolation of the total cellular mRNA was performed using the GeneJET RNA purification kit (Thermo Fisher Scientific) following manufacturer's instructions. Total RNA was purified with DNase and incubated at $37^{\circ} \mathrm{C}$ for 30 minutes using a thermocycler (Bio-Rad, Hercules, CA, USA). cDNA was synthesized using iScript kit (Bio-Rad). qRT-PCR was performed by using SsoAdvanced Universal SYBR Green Supermix Kit (Bio-Rad) on CFX96 Real-Time System (Bio-Rad). A $10 \mu \mathrm{L}$ reaction was used which included $5 \mu \mathrm{L}$ Sybr green, $3 \mu \mathrm{L}$ of distilled water, $0.5 \mu \mathrm{L}$ of forward primer, $0.5 \mu \mathrm{L}$ of reverse primer, and $1 \mu \mathrm{L}$ of 1:10 diluted cDNA. Each reaction was exposed to the following conditions: $98^{\circ} \mathrm{C}$ for 10 minutes, followed by 30 seconds of $98^{\circ} \mathrm{C}, 20$ seconds of $60^{\circ} \mathrm{C}$, and 30 seconds of $72^{\circ} \mathrm{C}$ for 44 cycles in 96-well optical reaction plates (Bio-Rad). Species specific reference genes were used to normalize the targeted genes. Primer sequences are listed in Additional file 1.

\section{Experimental design and statistical analysis}

Data are presented as the mean \pm standard error of the mean (SEM) of triplicates per analysis. Results with ${ }^{* *} p \leq 0.01$ were considered statistically significant. All analyses were performed using SPSS version 26 (SPSS Inc. USA). Statistical analysis were carried out by using one-way analysis of variance (ANOVA) test for multiple comparisons followed by post hoc tests and ANOVA for repeated measures followed by Tukey's post-hoc analysis.

\section{Results}

Differentiation and characterization of NSCs-derived from primitive MSCs. We first differentiated the primitive MSCs into NSCs (18), which displayed typical neural extension morphology, significant loss of MSC markers and increased expression of the neural genes, NESTIN, TUJ1, VIMENTIN, and PAX6, as well as neurotrophic factors, CNTF, BMP2, PDGF, BDNF, GDNF, IGF, EGF, and FGF (Fig. 1A-1E and Additional file 2). They also expressed neural proteins, NESTIN, TUJ1, VIMENTIN, and PAX6 (Fig. 1F-1I and Additional file 2). This protocol reproducibly yielded 73\% differentiation of MSCs into NSCs (Fig. 1J). 
Effect of cell transplantation in EAE mice. We then investigated the effects of MSCs and NSCs on the disease process in EAE mice. EAE was induced by myelin oligodendrocyte glycoprotein (MOG) peptide immunization in animals using an established protocol (16) and disease progression was followed by neurobehavioral analysis (Fig. 2A) (19). The first signs of EAE induction in animals were noticed 11-12 days after MOG injections. Then EAE stage 1 and 2 animals were intravenously injected with PKH26 labeled $10^{6}$ cells and animals were evaluated for neurobehavioral changes twice a day until they were sacrificed for post-transplantation analysis. Neurobehavioral analyses results showed that average clinical score of the EAE control $(4.5 \pm 0.5)$ was significantly higher than the average score of mice transplanted with MSCs $(1.7 \pm 0.3)$ and NSCs $(0.8 \pm 0.1)$ at stage 1 and MSCs $(2.4 \pm 0.2)$ and NSCs $(2.2 \pm$ 0.1 ) at stage 2 (Fig. $2 \mathrm{~B}$ and Additional file 3 ). In addition, the average weight of the untreated EAE control $(13 \mathrm{~g} \pm 0.6 \mathrm{~g})$ was significantly lower than the average weights of the mice transplanted with MSCs $(15.7 \mathrm{~g}$ $\pm 0.7 \mathrm{~g})$ and NSCs $(16 \mathrm{~g} \pm 0.4 \mathrm{~g})$ at stage 1 and MSCs $(14 \mathrm{~g} \pm 0.7 \mathrm{~g})$ and NSCs $(14.6 \mathrm{~g} \pm 0.7 \mathrm{~g})$ at stage 2 (Fig. $2 \mathrm{C}$ and Additional file 3 ). When MSCs were transplanted at EAE stage 1, they slowed and halted the disease progression. However, NSCs not only slowed but also reversed the disease process, and animal conditions were improved to near normal levels. Overall, NSCs showed greater efficacy than MSCs when transplanted at EAE stage 1 than stage 2. Animals were sacrificed two weeks post-transplantation, and blood, lungs, lymphatic and CNS tissues were collected for histological, biochemical, immunological and molecular analyses.

Homing of transplanted cells to the blood, lymphatic, and CNS systems. The labeled transplanted cells homed to the blood, spleen, brain, and spinal cord (Fig. 3A). Only an insignificant number of transplanted cells were found in the lungs of all animals, suggesting minimal lung trapping. Interestingly, significantly more transplanted MSCs (10.0\%) than NSCs (6.7\%) were observed in the blood of healthy animals, suggesting greater survivability of MSCs in the vascular system. In the blood of EAE stage 1 animals, the number of transplanted MSCs and NSCs was $9.3 \%$ and $10.3 \%$, respectively. Strikingly, a very low number of MSCs and NSCs (1.2\% and 2.0\%, respectively) were found in the blood of EAE stage 2 animals. Nearly half of transplanted cells were found in the spleen in all animals. Importantly, a significant number of transplanted cells were present in the CNS of EAE animals but not in healthy controls. In the case of EAE stage 1 animals, a similar number of transplanted MSCs and NSCs were found in the brain $7.0 \%$ and $7.5 \%$, respectively) but significantly more NSCs than MSCs ( $10.5 \%$ and $12.3 \%$, respectively) were found in spinal cord. Overall, the number of transplanted labeled MSCs and NSCs found in the CNS of EAE stage 1 animals were more than Stage 2. Notably, NSCs were significantly more in the spinal cord than MSCs. Overall, NSCs exhibited greater survivability and/or proliferation in EAE stage 1 animals than MSCs.

\section{Improvement in the histopathology of the brain and spinal cord of EAE mice by transplanted cells. EAE}

induction mediates $T$ cell activation resulting in increased infiltrates and demyelination as well as permeation of the BBB (20). The brain and spinal cord (Fig. 3B and 3D) showed presence of cell infiltrates in EAE animals but not in heathy controls. The number of infiltrates in both brain and spinal cord were significantly decreased in EAE stage 1 compared to stage 2 animals treated with MSCs and NSCs. Furthermore, the LFB staining of the brain and spinal cord (Fig. 3C and 3E) was greatly improved in NSC 
treated animals compared to those treated with MSCs. The stain intensity was significantly increased in the cortex and spinal cord of EAE stage 1 (less in stage 2) animals treated with the cells suggesting endogenous remyelination. Taken together, these results suggest that transplanted NSCs not only reduced cell infiltrates, but also showed increased remyelination in the CNS at EAE stage 1 compared to the stage 2 .

Modulation of the immune response by transplanted cells. The main effector cells of cell mediated immune response in EAE and MS are Treg (CD4 + and CD25+) and Th17 (CD4 + and IL17A+) (20). We investigated the level of these cells in the blood, spleen, and CNS by flow cytometry. Treg cells were significantly reduced to $8.6 \%$ and $7.3 \%$ in EAE animals from $35.6 \%$ and $43.0 \%$ in healthy controls in both the blood and spleen (Fig. 4A). In contrast, Treg cells were substantially increased in EAE animals transplanted with cells in the blood and spleen. In EAE stage 1 animals treated with MSCs, Treg cells were $27.4 \%$ and $41.1 \%$ in the blood and spleen, respectively. Whereas in EAE stage 2 animals treated with MSCs, Treg cells were $15.8 \%$ and $37.5 \%$ in the blood and spleen, respectively. On the other hand, in EAE stage 1 animals treated with NSCs, Treg cells were $39.3 \%$ and $42.0 \%$ in the blood and spleen, respectively. In EAE stage 2 animals treated with NSCs, Treg cells were $22.3 \%$ and $42.6 \%$ in the blood and spleen, respectively. In conclusion, Treg cell numbers increased in animals treated with both MSCs and NSCs; however, the improvements were more significant in the case of NSCs and their efficiency of restoring Treg levels was greater in EAE stage 1 than stage 2 animals. In the case of the CNS, Treg levels were $14.6 \%$ and $22.6 \%$, respectively, in EAE animals. While cell transplantation did not affect the infiltrated Treg cells in the brain, Treg cells were significantly reduced to $14.8 \%$ and $10.0 \%$ in the spinal cord of EAE stage 1 animals treated with MSCs and NSCs, respectively. Cell transplantation in EAE stage 2 animals was less effective, as Treg cells reduced only to $17.0 \%$ in both MSCs and NSCs. Taken together, NSCs showed greater efficacy than MSCs in reducing Treg cells in the spinal cord in EAE stage 1 animals. It is known that Treg cells increase during the chronic late stages of EAE (21). So, reduced Treg levels in animals treated with cells could contribute to improved disease symptoms.

As expected, Th17 cells were significantly increased from $10.3 \%$ and $6.9 \%$ in the blood and spleen of healthy controls to $15.3 \%$ and $13.7 \%$ in EAE animals, respectively. When treated with MSCs, Th17 cell levels were not significantly affected in the blood (14.9\%) but were reduced to normal levels (10.1\%) in the spleen in EAE stage 1 animals; however, no effect was noticed in the case of EAE stage 2 (Fig. 4A). Importantly, NSC transplantation reduced Th17 in EAE stage 1 and stage 2 animals to levels similar to the healthy control animals. Both the brain and spinal cord of EAE animals had significantly high levels of Th17 cells ( $22.5 \%$ and $30.5 \%$, respectively) as compared healthy control animals $(1.6 \%$ and $0.9 \%$, respectively). Upon MSC transplantation, Th17 cells were significantly reduced in the brain and spinal cord of EAE stage 1 animals (12.6\% and 20.3\%, respectively) but not in EAE stage 2 animals. On the other hand, NSC transplantation had a more prominent effect on reducing Th17 cells in both the brain and spinal cord in EAE stage 1 (11.5\% and $13.7 \%$, respectively) and in stage 2 (19.4\% and $22.2 \%$, respectively) animals. However, the NSC effect was less significant in EAE stage 2 animals. 
Effect of transplanted cells on immune infiltrates in the CNS. To investigate the effect of transplanted cells on the inflammatory response in EAE mice, we analyzed the expression of CD45, CD68, and CD3E markers, representing leukocytes, macrophages, and $\mathrm{T}$ cells, respectively. The results depicted in Fig. 4B to $4 \mathrm{D}$ show high expression of the markers in the brain and spinal cord of EAE animals but not in healthy controls. Importantly, MSCs and NSCs were more effective in reducing the expression of all three markers in the spinal cord of EAE stage 1 but not stage 2 animals. Clearly, NSCs exert a greater anti-inflammatory response, particularly in EAE stage 1 animals potentially by reducing the pro-inflammatory cells, macrophages, leukocytes, and T cells.

Restoration of myelination and inhibition of astrogliosis by MSCs and NSCs. We next examined the effect of transplanted cells on the proteins associated with myelination and neural regeneration. We observed a significant decrease in the expression of MOG, MBP, TUJ1, and NESTIN in the brain of EAE animals (Fig. 5A and 5C). The expression of MOG and MBP was significantly increased in the brain upon transplantation of NSCs in both stages of EAE. However, MSCs had a significant increase in EAE stage 1 animals only. Similarly, the expression of TUJ1 was significantly increased in the brain of both EAE stage 1 and stage 2 animals transplanted with NSCs, but this was only seen in MSC treated stage 1 animals. Upon induction of EAE, the expression of GFAP increased (Fig. 5A and 5C), due to increased astrogliosis in the brain of EAE animals (22). However, GFAP expression was reduced in both EAE stage 1 and stage 2 animals transplanted with cells. The trend in the expression of MOG, MBP, TUJ1, and NESTIN in the spinal cord of EAE animals treated with cells was similar to the brain. However, NESTIN was significantly increased in EAE stage 1 animals treated with NSCs and the decrease of GFAP in the spinal cord of transplanted animals was not significant (Fig. 5B and 5D). Overall, NSCs promoted greater remyelination and neural recovery as well as reduction of astrogliosis in the EAE stage 1 animals.

\section{Effect of cells on the transcription for inflammatory, astrogliosis, neuroprotection, and myelination genes.}

To determine the effects of transplanted cells on the expression of inflammatory, neuroprotection, and myelination genes, transcriptional analysis of the spleen, brain, and spinal cord tissues was performed. As expected, higher expression of the inflammatory markers, Cd3e, II-17a, II-2, II1 , II-6, Tnfa, and Ifny was observed in the brain, spinal cord, and spleen, respectively, in EAE animals compared to the healthy controls (Fig. 6A and 6B, Additional file 4, and Additional file 5). Expression of these markers was significantly reduced in the brain of MSC and NSC treated EAE stage 1 and stage 2 animals, except II-17a in MSC treated Stage 2 animals. In contrast, expression of the inflammatory markers were significantly reduced in the spinal cord when NSCs and MSCs were transplanted at EAE stage 1 but not stage 2 . Similar to the brain, expression of these markers was significantly reduced in the spleen of animals transplanted with both types of cells at EAE stage 1 and stage 2, except for the Cd3e, II-17a, II-2, and II-1 $\beta$ markers, in MSCs transplanted in EAE stage 2 animals.

Cytokine storm is elicited in MS. Although many cytokines take part in the "cytokine storm", IL-6 plays a crucial role. Dysregulated continual release of IL-6 has pleiotropic effects on chronic inflammation and autoimmunity, and also lead to the onset or development of various diseases including MS, acute respiratory distress syndrome, and multiple organ dysfunction syndrome (23). In EAE, IL-6 aggravates 
clinical symptoms and spinal cord pathology mainly by promoting pathogenic Th17 cells, which initiate and perpetuate inflammation and demyelination (24-26). IL-6 also affects autoreactive effector T cells in MS. IL-6 signaling supports T effector cell resistance to regulation by Treg cells, which may contribute to disease aggravation (27). Our results show that cell transplantation was effective at countering the induction of II-6 gene expression in spleen and CNS tissues. In addition, NSCs showed more efficacy in regulating Treg cells when compared to MSCs, suggesting that NSCs are more potent in eliciting antiinflammatory effects than MSCs in the CNS.

As anticipated, the expression of neural markers, Tuj1 and Nestin, was downregulated in the CNS in EAE animals but was significantly increased in transplanted animals (Fig. 6A and 6B and Additional file 5). Studies have shown that upregulation of Gfap and Ccnd1 is associated with reactivation of astrocytes (astrogliosis) $(22,28)$. Overall, EAE stage 1 animals transplanted with NSCs displayed reduction of astrogliosis genes in the brain and spinal cord. Our results also suggest that expression of Gfap and Ccdn 1 is likely to be regulated by the JAK/STAT pathway and promote the transcription of astrogliosis genes such as Gfap, Cntfra, and Nfl-b in EAE animals (Fig. 6A and 6B and Additional file 5). Our results demonstrated that there was an upregulation of these astrogliosis genes via activation of the JAK/STAT pathway leading to an increase of astrogliosis in EAE mice. However, in EAE stage 1 animals treated with NSCs, these genes were significantly downregulated causing a decrease in astrogliosis in the CNS.

We determined that several paracrine neurotrophic factors were significantly upregulated in NSCs versus MSCs in vitro (Fig. 1E). Predictably, these factors contributed to the greater efficacy of NSCs for neuroprotection and the reversal of EAE symptoms. The CNS also showed higher expression of several neurotrophic factors such as Igf, Bdnf, and Trkb, which are pertinent to neuroprotection (Fig. 6A and 6B and Additional file 5). The fact that the expression of these factors is improved more significantly in EAE animals transplanted with NSCs, and that the animals recovered to near normal provide a 'proof of concept' that the use of NSC therapy in MS patients would more effective than using MSCs.

Factors controlling myelination have been reported to synergistically induce the expression of Krox-20, which is involved in activation of myelin genes such as $M p z$ and $M b p(29,30)$. Our results showed that several genes, Erk2, Krox-20, Oct-6, Mpz, Mbp, and Mog, associated with myelination were downregulated in EAE animals. Expression of these genes was significantly increased in the brain and spinal cord of EAE stage 1 animals injected with both types of cells, but NSCs provided a greater improvement compared to EAE stage 2 (Fig. 6A and 6B and Additional file 5).

Mechanism of neural protection and regeneration. Our results showed downregulation in EAE animals but increased expression of Oct- 6 and Krox-20 in transplanted animals, which suggests that the MAPK pathway may be involved in the regulation of these genes, because inhibition of MAPK signaling has shown to upregulate myelination associated genes (31). Chronic demyelination favors neurodegeneration of denuded axons, which causes irreversible neuronal deficits and disability in MS patients (32). Furthermore, transplanted cells promoted neural survival and regeneration responsible for increased LFB stain intensity, indicative of an increase in remyelination of denuded axons. Taken together, results of this 
investigation suggest that NSCs influence not only MAPK but also several other signaling pathways, particularly MAPK, JAK/STAT, and PI3K/AKT as illustrated in Fig. 6C.

\section{Discussion}

In the current study, we used highly proliferative MSCs isolated from a novel region of the umbilical cord tissue $(9,10)$, and differentiated them into a large amount of NSCs required for animal studies. The results of behavioral analysis showed that NSCs were more efficacious than MSCs when injected into animals with an early stage of EAE. While MSCs only halted the progression of disease symptoms, NSCs not only slowed but also reversed the disease process. Reversal of disease progress was parallel with weight gain that is typically lost due to EAE.

Although the $\mathrm{PKH} 26$ is a lipophilic tracer, previous studies have shown that the label has been traceable for more than 8 weeks following injection $(17,33)$. In the current study, post-transplantation analysis showed that majority of the PKH26 labeled transplanted cells were found in the blood, spleen, and CNS in EAE stage 1 animals. However, there was a significant decrease of the number of cells found in the blood in EAE stage 2 animals transplanted with cells, possibly due to autophagy. It has been previously reported that regulation of autophagy plays an important role in providing neuroprotection via modulation of inflammatory and immune reaction in the CNS (34-36). Conceivably, cells transplanted at EAE stage 1 evaded autophagy induction, homed to the CNS and exhibited anti-inflammatory and neuroprotective characteristics.

In EAE mice, T cell activated cells travel through the BBB into the CNS leading to an increase of cell infiltrates and demyelination of CNS neurons (20). We also observed a significant increase of cell infiltrates in the brain and spinal cord of EAE animals as compared to the healthy control. However, when cells were transplanted at stage 1 , there was a significant decrease of infiltrate cells. This could be due to the anti-inflammatory properties of MSCs and NSCs leading to a significant reduction of infiltrates (37). However, NSCs displayed a greater effect in reducing the number of infiltrates in the CNS. In addition, EAE mice transplanted with cells at stage 1 also had a greater effect on remyelination of the neurons in the CNS compared to stage 2 mice. These results are in agreement with previous studies where pharmacological drugs promoted remyelination of spinal cord neurons (38). Our approach significantly restored the lost myelin to near healthy control levels in the CNS, specifically those transplanted with NSCs at stage 1.

Studies have shown that Treg and Th17 play an important role in the development of EAE and the immune response correlated with the disease (39-41). Treg maintains cell immune tolerance, whereas Th17 mediates the inflammatory response under abnormal conditions such as during an injury (40). We observed an imbalance between Treg and Th17 in EAE animals similar to previous reports (40). At the onset of EAE, the levels of Treg decreased and Th17 increased in the blood and spleen. However, after NSC transplantation at EAE stage 1, the imbalance was corrected and the levels of Treg and Th17 resembled those found in the blood and spleen of the healthy controls. Similarly, levels of Treg and Th17 
were reduced in the CNS of animals transplanted with NSCs. The underlying mechanism for the restoration of the Treg/Th17 levels could be due to the transplanted cells modulating the function of $T$ cells by inhibiting the production of TNFa and IFNy, as reported previously (42-44). Therefore, we investigated the transcription of TNFa and IFNY in EAE mice transplanted with cells. As expected, we found a reduction of these pro-inflammatory markers in the spleen and CNS tissues of animals transplanted with cells, further confirming the regulation of Treg and Th17 by transplanted cells. More importantly, the anti-inflammatory effect was found to be more prominent in animals transplanted with cells at EAE stage 1. Furthermore, the activity of leukocytes, macrophages, and T cells in the CNS were also decreased in animals transplanted with cells at EAE stage 1. Again, NSCs provided a greater affect in the reduction of these infiltrates in the spinal cord.

In order to elucidate the mechanism that might explain the therapeutic effects of MSCs and NSCs in EAE mice, we investigated astrogliosis, neuroprotection, and remyelination in the CNS. It is known that in chronic EAE, lesions become inactive resulting in accumulation of reactive astrocyte (GFAP+) filaments in the CNS (45). We found that astrogliosis genes, Gfap and Ccnd1, were upregulated in EAE mice as well as GFAP + mouse cells, which were integrated into the brain and grey matter of the spinal cord. Upon cell transplantation, a significant decrease in the expression of astrogliosis genes in the CNS as well as a recession of GFAP + cells was found in the grey matter. These results agreed with previous reports showing the potential of MSCs and NSCs to reduce astrogliosis leading to an increase in neuroprotection and remyelination of neurons in the CNS $(42,46,47)$.

MSCs and NSCs have the capability of promoting neuroprotection by secreting paracrine factors (BDNF) that can trigger the repair pathways (i.e. PI3K/AKT) (48-50). Our results showed an upregulation of the neurotrophic factors in EAE mice when transplanted with MSCs and NSCs at EAE stage 1. However, at EAE stage 2, despite insignificant difference in the expression of neurotrophic factors, NSCs appeared to be more effective in providing neuroprotection than MSCs. These results correspond with the improvement of neurobehavior observed in the transplanted animals.

In MS, the demyelination is caused by myelin-specific T cells that target the myelin on neurons in the CNS (51).

After entering the CNS, these cells are reactivated by local and infiltrating activated antigen-presenting cells, resulting in subsequent inflammation, then demyelination and axonal damage.

Our results also demonstrated that NSCs promoted remyelination in the CNS as indicated by LFB staining as reported in previous studies $(52,53)$. Furthermore, upregulation of the genes involved in myelination, suggested that transplanted cells promoted remyelination in the CNS. Our results also suggest predictive mechanisms involved in the neuroprotection and remyelination of neuron via the activation of JAK/STAT, SMAD, MAPK, and PI3K pathways as depicted in Fig. 6C.

\section{Conclusion}


In brief, this study demonstrated the potential of NSCs to induce anti-inflammatory responses and provide neuroprotection as well as suggest promotion of endogenous neuroregeneration and remyelination of neurons denuded in EAE animals. Although, a number of clinical trials utilizing autologous bone marrow MSCs and umbilical cord derived MSCs, the results were not always conclusive. In some cases, there was improvement in the quality of life measures one month following treatment; however, the participants reported similar levels of pain one year after treatment $(54,55)$. Furthermore, most of these studies were limited to phase I and II trials due to limited availability of cells. Four clinical trials utilized bone marrow MSC derived NSCs, showing improvement of neurological disability (56). Two clinical trials used human fetal-derived NSCs (57); however, results of these phase I/II studies have not been reported. Again, these NSCs were limited in numbers their isolation from fetal tissue and use could pose ethical and moral concerns (58). While our results are highly promising, further studies should be pursued to determine if higher or multiple doses of cells injected at advanced stages of EAE could reverse the disease process. It would also be interesting to know if the cell treatment at the time of MOG injection could block induction of EAE in mice. Nevertheless, our study provided proof of the concept and basis for investigating the efficacy of NSCs derived from primitive MSCs to treat MS in clinical trials.

\section{Abbreviations}

\section{BBB}

Blood brain barrier

bFGF

Basic fibroblast growth factor

\section{CNS}

Central nervous system

\section{EAE}

Experimental autoimmune encephalomyelitis

\section{EGF}

Epidermal growth factor

\section{FACS}

Fluorescence-activated cell sorting

\section{FBS}

Fetal bovine serum

\section{GM}

Growth medium

\section{GvHD}

Graft verse host disease

\section{H\&E}

Hematoxylin and eosin

\section{i.p}

Intraperitoneally 
IACUC

Institutional Animal Care and Use Committee

IBC

Institutional Biosafety Committee

LFB

Luxol fast blue

MHC

Major histocompatibility complex

MOG

Myelin oligodendrocyte glycoprotein

MS

Multiple sclerosis

MSCs

Mesenchymal stem cells

NSCs

Neural stem cells

qRT-PCR

Quantitative reverse transcriptase polymerase chain reaction

SEM

Standard error of the mean

\section{Declarations}

\section{Ethics approval and consent to participate}

All procedures and handling of animals during the investigations were reviewed and approved by the Institutional Animal Care and Use Committee (IACUC \#18081) and the Institutional Biosafety Committee (IBC \#2858) at Oakland University.

\section{Consent for publication}

Not applicable.

\section{Availability of data and materials}

All data generated or analyzed during this study are included in this published article and its supplementary information files.

\section{Competing interests}

The authors declare that they have no competing interests. 
Funding: The study was supported by the OU-WB Institute for Stem Cell and Regenerative Medicine (ISCRM), Oakland University, Rochester, MI. C. Brown and C. McKee received the Provost Graduate Research Awards from Oakland University for this project.

\section{Authors' contributions}

Conceptualization: G.R.C., C.B., and C.M.; Methodology: G.R.C., C.B., C.M., S.H., S.K., and D.L.F.; Software: C.B. and C.M.; Validation: C.B., C.M., D.L.F., and G.R.C.; Formal Analysis: C.B., C.M., D.L.F., and G.R.C.; Investigation: C.B., C.M., S.H., S.K., D.L.F, and G.R.C.; Resources: G.R.C.; Data curation: C.B., C.M., and G.R.C.; Supervision: G.R.C.; Visualization: C.B., C.M., S.H., S.K., D.L.F., and G.R.C.; Funding acquisition: G.R.C.; Project administration: G.R.C.; Writing - original draft: C.B. and G.R.C.; Writing - review \& editing: all authors.

\section{Acknowledgements}

We thank C. Govind and D. Svinarich for fruitful discussion and for thoroughly reviewing the manuscript.

\section{References}

1. Jančić J, Nikolić B, Ivančević N, Henčić B, Samardžić J. Multiple Sclerosis Therapies in Pediatric Patients: Challenges and Opportunities. In: Zagon IS, McLaughlin PJ, editors. Multiple Sclerosis: Perspectives in Treatment and Pathogenesis. Brisbane (AU): Codon Publications Copyright: The Authors.; 2017.

2. Dulamea AO. Role of Oligodendrocyte Dysfunction in Demyelination, Remyelination and Neurodegeneration in Multiple Sclerosis. Advances in experimental medicine and biology. 2017;958:91-127.

3. Owens GM. Economic burden of multiple sclerosis and the role of managed sare organizations in multiple sclerosis management. Am J Manag Care. 2016;22(6 Suppl):s151-s8.

4. Xiao J, Yang R, Biswas S, Zhu Y, Qin X, Zhang M, et al. Neural Stem Cell-Based Regenerative Approaches for the Treatment of Multiple Sclerosis. Mol Neurobiol. 2018;55(4):3152-71.

5. Villoslada P, Steinman L. New targets and therapeutics for neuroprotection, remyelination and repair in multiple sclerosis. Expert opinion on investigational drugs. 2020;29(5):443-59.

6. Genc B, Bozan HR, Genc S, Genc K. Stem Cell Therapy for Multiple Sclerosis. Advances in experimental medicine and biology. 2019;1084:145-74.

7. Fontaine MJ, Shih H, Schäfer R, Pittenger MF. Unraveling the Mesenchymal Stromal Cells' Paracrine Immunomodulatory Effects. Transfusion medicine reviews. 2016;30(1):37-43.

8. Brown C, McKee C, Bakshi S, Walker K, Hakman E, Halassy S, et al. Mesenchymal stem cells: Cell therapy and regeneration potential. Journal of Tissue Engineering and Regenerative Medicine. 2019;13(9):1738-55. 
9. Beeravolu N, Khan I, McKee C, Dinda S, Thibodeau B, Wilson G, et al. Isolation and comparative analysis of potential stem/progenitor cells from different regions of human umbilical cord. Stem cell research. 2016;16(3):696-711.

10. Beeravolu N, McKee C, Alamri A, Mikhael S, Brown C, Perez-Cruet M, et al. Isolation and Characterization of Mesenchymal Stromal Cells from Human Umbilical Cord and Fetal Placenta. Journal of visualized experiments : JoVE. 2017(122).

11. Liu L, Eckert MA, Riazifar H, Kang DK, Agalliu D, Zhao W. From blood to the brain: can systemically transplanted mesenchymal stem cells cross the blood-brain barrier? Stem Cells International 2013;2013:435093.

12. Aboody KS, Brown A, Rainov NG, Bower KA, Liu S, Yang W, et al. Neural stem cells display extensive tropism for pathology in adult brain: evidence from intracranial gliomas. Proc Natl Acad Sci U S A. 2000;97(23):12846-51.

13. Imitola J, Raddassi K, Park KI, Mueller FJ, Nieto M, Teng YD, et al. Directed migration of neural stem cells to sites of CNS injury by the stromal cell-derived factor 1alpha/CXC chemokine receptor 4 pathway. Proc Natl Acad Sci U S A. 2004;101(52):18117-22.

14. Snyder EY, Yoon C, Flax JD, Macklis JD. Multipotent neural precursors can differentiate toward replacement of neurons undergoing targeted apoptotic degeneration in adult mouse neocortex. Proc Natl Acad Sci U S A. 1997;94(21):11663-8.

15. Glatigny S, Bettelli E. Experimental Autoimmune Encephalomyelitis (EAE) as Animal Models of Multiple Sclerosis (MS). Cold Spring Harb Perspect Med. 2018;8(11).

16. Kuerten S, Kostova-Bales DA, Frenzel LP, Tigno JT, Tary-Lehmann M, Angelov DN, et al. MP4- and MOG:35-55-induced EAE in C57BL/6 mice differentially targets brain, spinal cord and cerebellum. J Neuroimmunol. 2007;189(1-2):31-40.

17. Beeravolu N, Brougham J, Khan I, McKee C, Perez-Cruet M, Chaudhry GR. Human umbilical cord derivatives regenerate intervertebral disc. J Tissue Eng Regen Med. 2018;12(1):e579-e91.

18. Bakshi S, McKee C, Walker K, Brown C, Chaudhry GR. Toxicity of JQ1 in neuronal derivatives of human umbilical cord mesenchymal stem cells. Oncotarget. 2018;9(73):33853-64.

19. Dupree JL, Feinstein DL. Influence of diet on axonal damage in the EAE mouse model of multiple sclerosis. J Neuroimmunol. 2018;322:9-14.

20. Fletcher JM, Lalor SJ, Sweeney CM, Tubridy N, Mills KHG. T cells in multiple sclerosis and experimental autoimmune encephalomyelitis. Clinical \& Experimental Immunology. 2010;162(1):111.

21. Duffy SS, Keating BA, Perera CJ, Lees JG, Tonkin RS, Makker PGS, et al. Regulatory T Cells and Their Derived Cytokine, Interleukin-35, Reduce Pain in Experimental Autoimmune Encephalomyelitis. The Journal of neuroscience : the official journal of the Society for Neuroscience. 2019;39(12):2326-46.

22. Moreno M, Guo F, Mills Ko E, Bannerman P, Soulika A, Pleasure D. Origins and significance of astrogliosis in the multiple sclerosis model, MOG peptide EAE. J Neurol Sci. 2013;333(1-2):55-9. 
23. Tanaka T, Narazaki M, Kishimoto T. IL-6 in inflammation, immunity, and disease. Cold Spring Harbor perspectives in biology. 2014;6(10):a016295-a.

24. Samoilova EB, Horton JL, Hilliard B, Liu TS, Chen Y. IL-6-deficient mice are resistant to experimental autoimmune encephalomyelitis: roles of IL- 6 in the activation and differentiation of autoreactive T cells. Journal of immunology (Baltimore, Md : 1950). 1998;161(12):6480-6.

25. Hu Z, Cui Y, Qiao X, He X, Li F, Luo C, et al. Silencing miR-150 Ameliorates Experimental Autoimmune Encephalomyelitis. Frontiers in neuroscience. 2018;12:465-.

26. Zager A, Peron JP, Mennecier G, Rodrigues SC, Aloia TP, Palermo-Neto J. Maternal immune activation in late gestation increases neuroinflammation and aggravates experimental autoimmune encephalomyelitis in the offspring. Brain, behavior, and immunity. 2015;43:159-71.

27. Schneider A, Long SA, Cerosaletti K, Ni CT, Samuels P, Kita M, et al. In active relapsing-remitting multiple sclerosis, effector $\mathrm{T}$ cell resistance to adaptive $\mathrm{T}$ (regs) involves IL-6-mediated signaling. Science translational medicine. 2013;5(170):170ra15.

28. Zamanian JL, Xu L, Foo LC, Nouri N, Zhou L, Giffard RG, et al. Genomic analysis of reactive astrogliosis. The Journal of neuroscience : the official journal of the Society for Neuroscience. 2012;32(18):6391-410.

29. Mirsky R, Woodhoo A, Parkinson DB, Arthur-Farraj P, Bhaskaran A, Jessen KR. Novel signals controlling embryonic Schwann cell development, myelination and dedifferentiation. Journal of the peripheral nervous system : JPNS. 2008;13(2):122-35.

30. Parkinson DB, Bhaskaran A, Droggiti A, Dickinson S, D'Antonio M, Mirsky R, et al. Krox-20 inhibits Jun-NH2-terminal kinase/c-Jun to control Schwann cell proliferation and death. The Journal of cell biology. 2004;164(3):385-94.

31. Liu X, Zhao Y, Peng S, Zhang S, Wang M, Chen Y, et al. BMP7 retards peripheral myelination by activating p38 MAPK in Schwann cells. Scientific Reports. 2016;6:31049.

32. Lucchinetti C, Brück W, Parisi J, Scheithauer B, Rodriguez M, Lassmann H. Heterogeneity of multiple sclerosis lesions: implications for the pathogenesis of demyelination. Ann Neurol. 2000;47(6):70717.

33. Perez-Cruet M, Beeravolu N, McKee C, Brougham J, Khan I, Bakshi S, et al. Potential of Human Nucleus Pulposus-Like Cells Derived From Umbilical Cord to Treat Degenerative Disc Disease. Neurosurgery. 2019;84(1):272-83.

34. François A, Terro F, Quellard N, Fernandez B, Chassaing D, Janet T, et al. Impairment of autophagy in the central nervous system during lipopolysaccharide-induced inflammatory stress in mice. Molecular brain. 2014;7:56.

35. Shao BZ, Wei W, Ke P, Xu ZQ, Zhou JX, Liu C. Activating cannabinoid receptor 2 alleviates pathogenesis of experimental autoimmune encephalomyelitis via activation of autophagy and inhibiting NLRP3 inflammasome. CNS neuroscience \& therapeutics. 2014;20(12):1021-8.

36. Shao BZ, Ke P, Xu ZQ, Wei W, Cheng MH, Han BZ, et al. Autophagy Plays an Important Role in Antiinflammatory Mechanisms Stimulated by Alpha7 Nicotinic Acetylcholine Receptor. Frontiers in 
immunology. 2017;8:553.

37. Farinazzo A, Angiari S, Turano E, Bistaffa E, Dusi S, Ruggieri S, et al. Nanovesicles from adiposederived mesenchymal stem cells inhibit T lymphocyte trafficking and ameliorate chronic experimental autoimmune encephalomyelitis. Scientific reports. 2018;8(1):7473-.

38. Thompson KK, Nissen JC, Pretory A, Tsirka SE. Tuftsin Combines With Remyelinating Therapy and Improves Outcomes in Models of CNS Demyelinating Disease. Frontiers in immunology. 2018;9:2784-.

39. Zheng Q, Yang T, Fang L, Liu L, Liu H, Zhao H, et al. Effects of Bu Shen Yi Sui Capsule on Th17/Treg cytokines in C57BL/6 mice with experimental autoimmune encephalomyelitis. BMC complementary and alternative medicine. 2015;15:60.

40. Lu P, Cao Y, Wang M, Zheng P, Hou J, Zhu C, et al. Mature dendritic cells cause Th17/Treg imbalance by secreting TGF- $\beta 1$ and IL- 6 in the pathogenesis of experimental autoimmune encephalomyelitis. Cent Eur J Immunol. 2016;41(2):143-52.

41. Kleinewietfeld M, Hafler DA. The plasticity of human Treg and Th17 cells and its role in autoimmunity. Semin Immunol. 2013;25(4):305-12.

42. Bai L, Lennon DP, Eaton V, Maier K, Caplan Al, Miller SD, et al. Human bone marrow-derived mesenchymal stem cells induce Th2-polarized immune response and promote endogenous repair in animal models of multiple sclerosis. Glia. 2009;57(11):1192-203.

43. Xin Y, Gao J, Hu R, Li H, Li Q, Han F, et al. Changes of immune parameters of T lymphocytes and macrophages in EAE mice after BM-MSCs transplanation. Immunology letters. 2020.

44. Kurte M, Bravo-Alegria J, Torres A, Carrasco V, Ibanez C, Vega-Letter AM, et al. Intravenous administration of bone marrow-derived mesenchymal stem cells induces a switch from classical to atypical symptoms in experimental autoimmune encephalomyelitis. Stem Cells Int. 2015;2015:140170.

45. Brambilla R. The contribution of astrocytes to the neuroinflammatory response in multiple sclerosis and experimental autoimmune encephalomyelitis. Acta Neuropathol Commun. 2019;137(5):757-83.

46. Bravo B, Gallego MI, Flores Al, Bornstein R, Puente-Bedia A, Hernández J, et al. Restrained Th17 response and myeloid cell infiltration into the central nervous system by human decidua-derived mesenchymal stem cells during experimental autoimmune encephalomyelitis. Stem cell research \& therapy. 2016;7:43-.

47. Pluchino S, Quattrini A, Brambilla E, Gritti A, Salani G, Dina G, et al. Injection of adult neurospheres induces recovery in a chronic model of multiple sclerosis. Nature. 2003;422(6933):688-94.

48. Xu L, Xing Q, Huang T, Zhou J, Liu T, Cui Y, et al. HDAC1 Silence Promotes Neuroprotective Effects of Human Umbilical Cord-Derived Mesenchymal Stem Cells in a Mouse Model of Traumatic Brain Injury via PI3K/AKT Pathway. Front Cell Neurosci. 2019;12:498-.

49. Koh SH, Lo EH. The Role of the PI3K Pathway in the Regeneration of the Damaged Brain by Neural Stem Cells after Cerebral Infarction. J Clin Neurol. 2015;11(4):297-304. 
50. Peltier J, O'Neill A, Schaffer DV. PI3K/Akt and CREB regulate adult neural hippocampal progenitor proliferation and differentiation. Developmental neurobiology. 2007;67(10):1348-61.

51. Goverman J. Autoimmune T cell responses in the central nervous system. Nat Rev Immunol. 2009;9(6):393-407.

52. Maeda Y, Nakagomi N, Nakano-Doi A, Ishikawa H, Tatsumi Y, Bando Y, et al. Potential of Adult Endogenous Neural Stem/Progenitor Cells in the Spinal Cord to Contribute to Remyelination in Experimental Autoimmune Encephalomyelitis. Cells. 2019;8(9):1025.

53. Inoue M, Honmou O, Oka S, Houkin K, Hashi K, Kocsis JD. Comparative analysis of remyelinating potential of focal and intravenous administration of autologous bone marrow cells into the rat demyelinated spinal cord. Glia. 2003;44(2):111-8.

54. Alghwiri AA, Jamali F, Aldughmi M, Khalil H, Al-Sharman A, Alhattab D, et al. The effect of stem cell therapy and comprehensive physical therapy in motor and non-motor symptoms in patients with multiple sclerosis: A comparative study. Medicine (Baltimore). 2020;99(34):e21646-e.

55. Riordan NH, Morales I, Fernández G, Allen N, Fearnot NE, Leckrone ME, et al. Clinical feasibility of umbilical cord tissue-derived mesenchymal stem cells in the treatment of multiple sclerosis. $\mathrm{J}$ Transl Med. 2018;16(1):57.

56. Harris VK, Stark J, Vyshkina T, Blackshear L, Joo G, Stefanova V, et al. Phase I Trial of Intrathecal Mesenchymal Stem Cell-derived Neural Progenitors in Progressive Multiple Sclerosis. EBioMedicine. 2018;29:23-30.

57. ClinicalTrials.gov. US National library of medicine. 2020.

58. Ishii T, Eto K. Fetal stem cell transplantation: Past, present, and future. World journal of stem cells. 2014;6(4):404-20.

\section{Figures}


A

MSCs NSCs

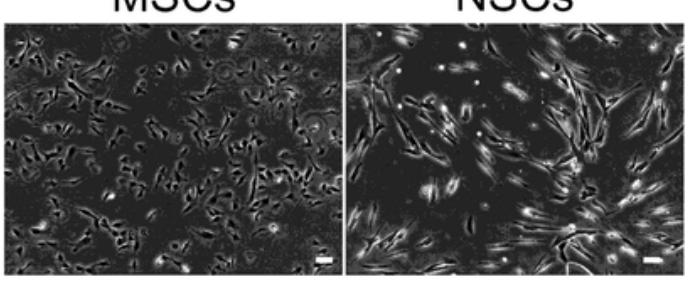

B Unstained-APC Unstained-FITC CD29-APC
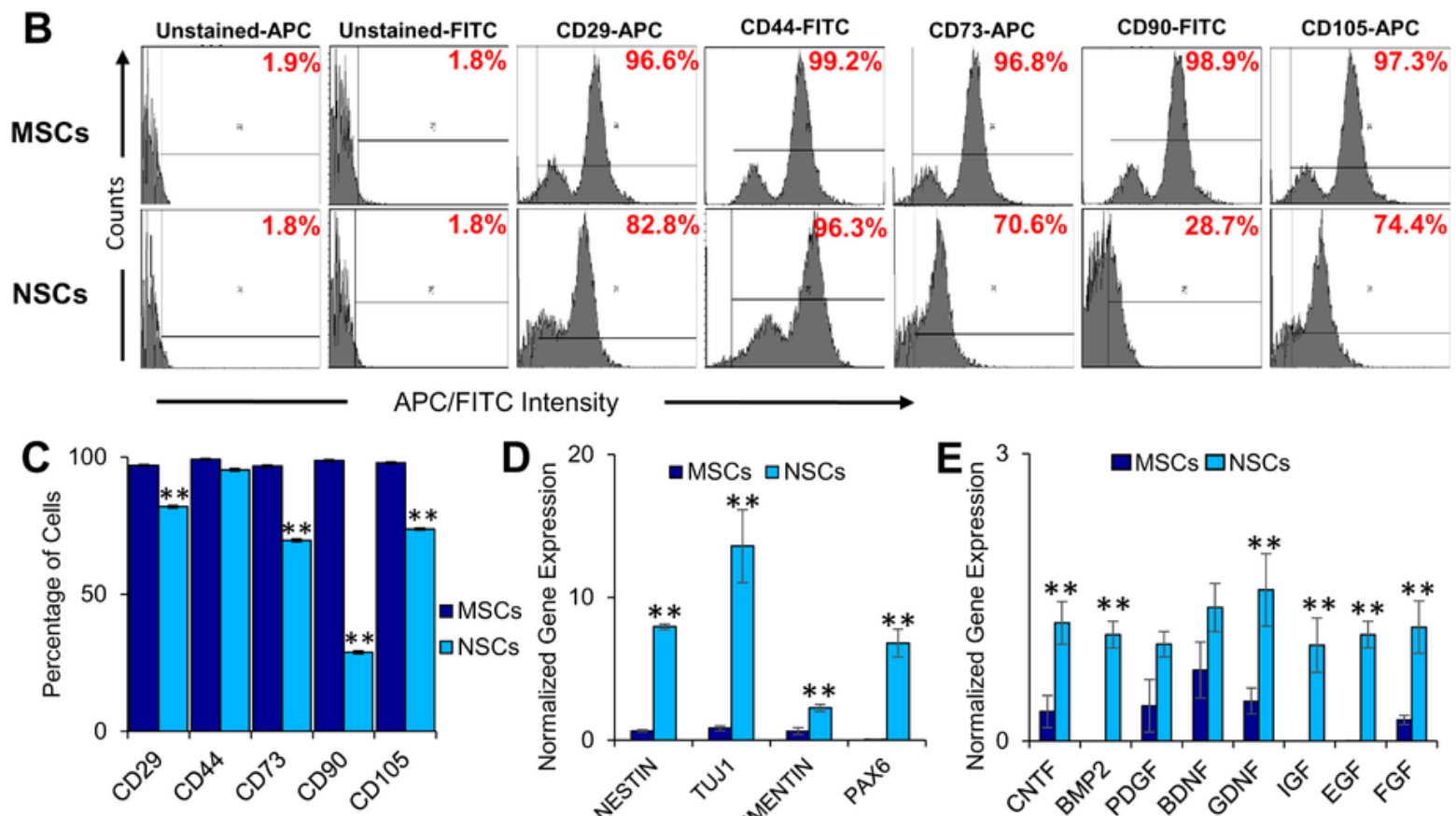

$\left.\mathbf{D}_{\varsigma_{\bar{\delta}}}{ }^{20}\right] \quad$-mSCs anSCs

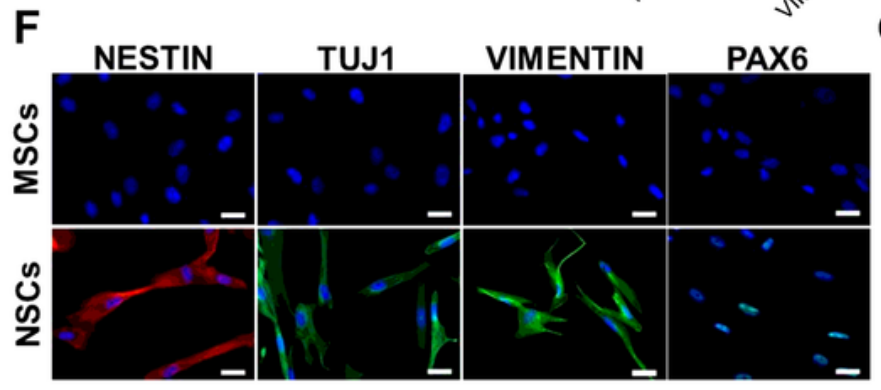

G
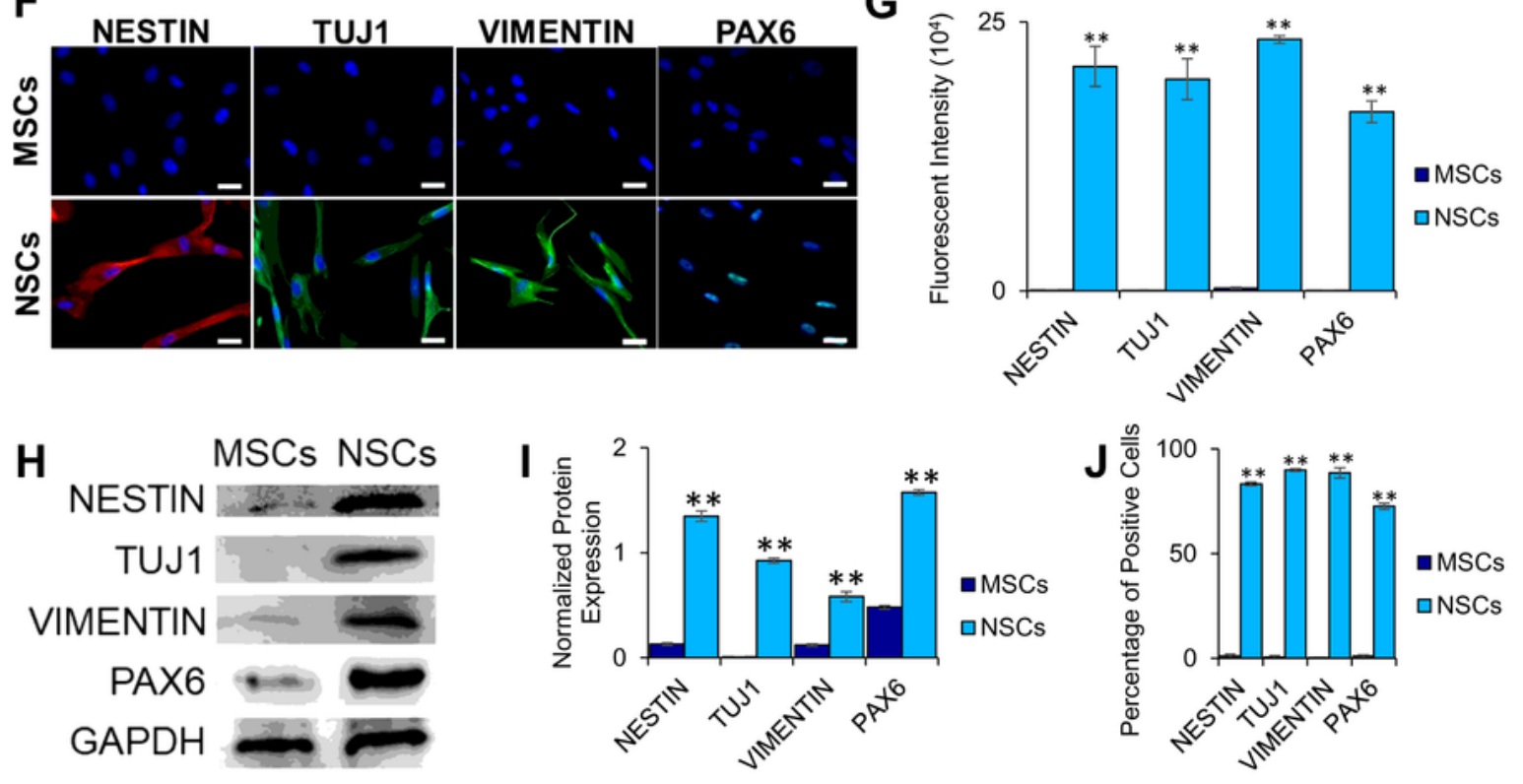

Figure 1

Differentiation and characterization of NSCs derived from primitive MSCs. (A) Phase contrast images of the primitive MSCs and NSCs. Scale bars represent $100 \mu \mathrm{m}$ (magnification: 4x). (B and C) Histograms and graphical representation of expression of mesenchymal markers in primitive MSCs and NSCs as determined by flow cytometry. All experiments were carried out in triplicate. (D) Expression of neural specific genes in the differentiated cells as determined by qRT-PCR. Gene expression was normalized to 
GAPDH and ACTIN and error bars represent the SEM of triplicate experiments ( $\left.{ }^{\star *} p \leq 0.01\right)$. (E) Neurotrophic gene expression was determined by qRT-PCR. Gene expression was normalized to GAPDH and ACTIN and error bars represent the SEM of triplicate experiments ( $\left.{ }^{*} p \leq 0.01\right)$. ( $F$ ) Expression of neural specific proteins was visualized by immunocytostaining. Shown are merge images of DAPI (blue) and human antibodies (green and red). All scale bars represent $100 \mu \mathrm{m}$ (Magnification: 10x). (G) Measurement of fluorescent intensity of proteins immunocytostained ( $\left.{ }^{\star \star} p \leq 0.01\right)$. (H) Western blot analysis of neural specific proteins (I) Quantitative analysis of normalized protein expression using Image $\mathrm{J}$ software ( ${ }^{\star \star} \mathrm{p} \leq 0.01$ ). All proteins were normalized to GAPDH expression. $(\mathrm{J})$ Percentage of positive cells immunocytostained (**p $\leq 0.01)$. 


\begin{tabular}{|l|l|}
\hline Acore & Symptoms \\
\hline 0 & No clinical signs \\
\hline 0.5 & Partial limp tail \\
\hline 1 & Limp tail \\
\hline 1.5 & Mild impaired righting \\
\hline 2 & Severe impaired righting \\
\hline 2.5 & Mild paresis of one hind limb \\
\hline 3 & Severe paresis of one hind limb \\
\hline 3.5 & $\begin{array}{l}\text { Severe paresis of one hind limb and mild } \\
\text { paresis of second hind limb }\end{array}$ \\
\hline 4 & Paresis of two hind limbs \\
\hline 4.5 & $\begin{array}{l}\text { Paresis of both hind limb and partial fore } \\
\text { limb }\end{array}$ \\
\hline 5 & Severe paralysis or death \\
\hline
\end{tabular}

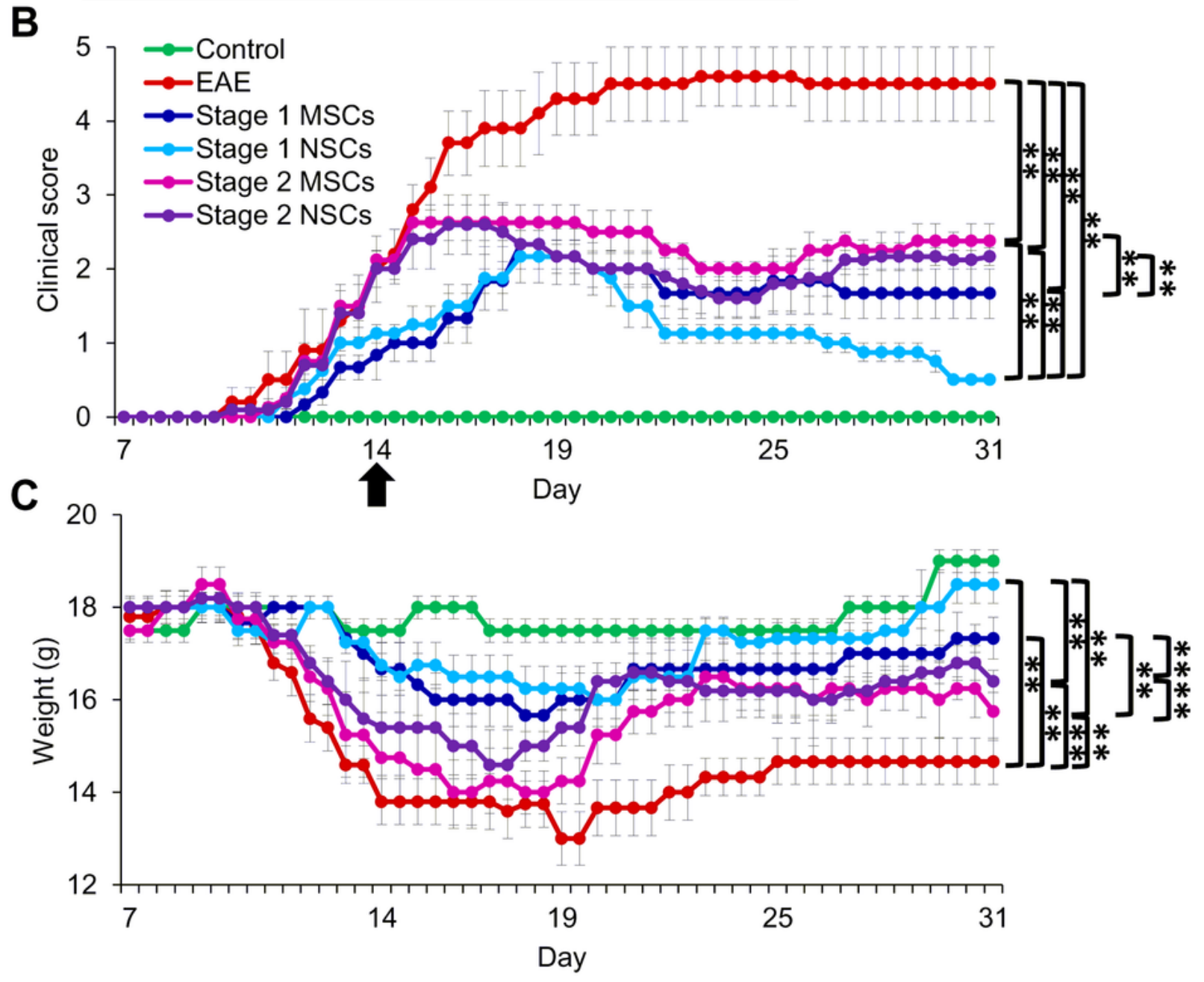

Figure 2

Effect of cell transplantation on EAE clinical symptoms and scoring. (A) Clinical EAE score scale. Averages ( $n=6$ each) of (B) clinical score of disease progression (stage 0-5) and (C) weight of mice following MOG immunization in comparison to non-EAE induced mice (control). Primitive MSCs and NSCs were transplanted on day 14 (indicated by black arrow) when mice displayed a clinical score of stage 1 or $2\left({ }^{\star *} p \leq 0.01\right)$. 


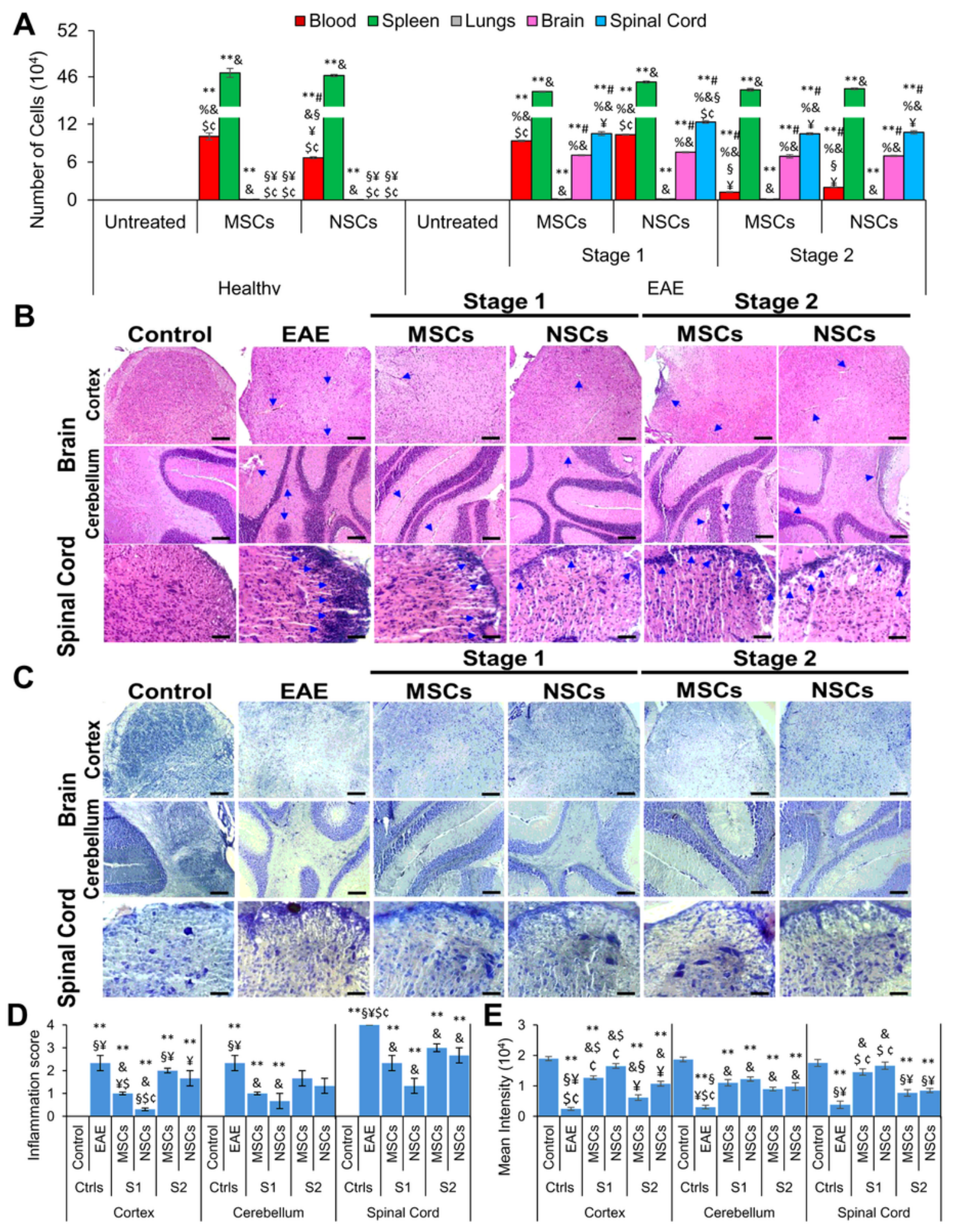

Figure 3

Analysis of various tissues from EAE mice transplanted with labeled cells. (A) Homing of transplanted MSCs and NSCs in the blood, spleen, lungs, brain, and spinal cord as determined by flow cytometry. Symbols, **, \#, \%, \&, §, ¥, \$, and $\zeta$ indicate significant difference at $p \leq 0.01$ between all experimental conditions: untreated healthy, healthy+MSCs, healthy+NSCs, untreated EAE, EAE stage $1+M S C s, E A E$ stage 1 + NSCs, EAE stage 2 + MSCs, and EAE stage 2 + NSCs, respectively. (B and C) Histological 
analysis of paraffin sections of the brain and spinal cord stained with H\&E and LFB to detect cellular infiltrates and myelination, respectively. Blue arrows indicate mouse infiltrates. Scale bars represent 50 $\mu m$ (magnification: 40x). (D and E) Inflammation score and LFB stain intensity of brain and spinal cord paraffin sections, respectively, representing various treatments.
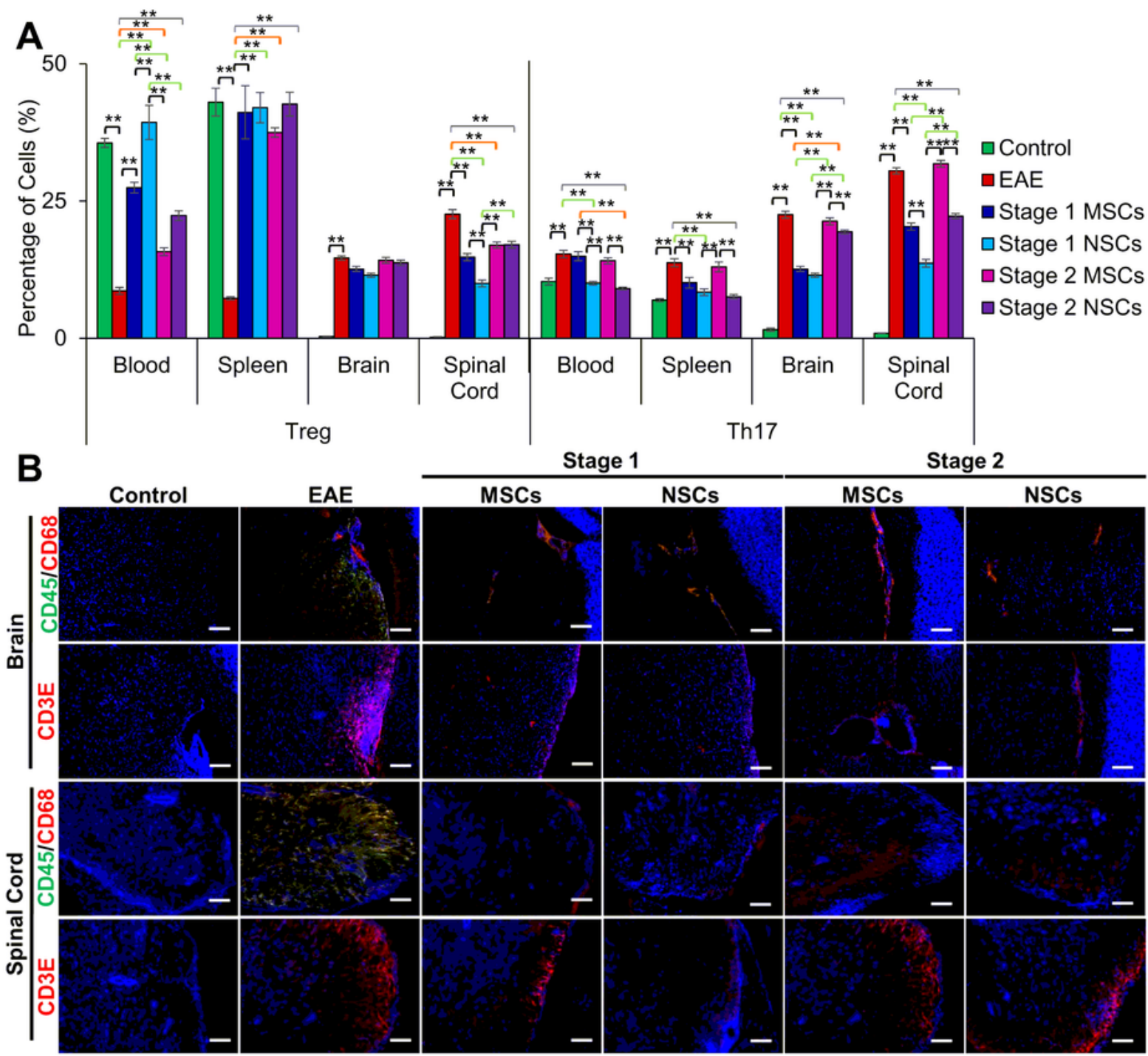

C

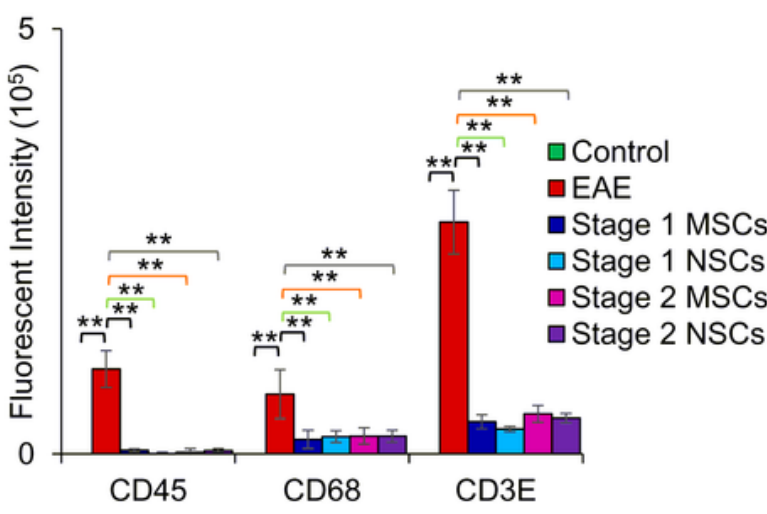

D

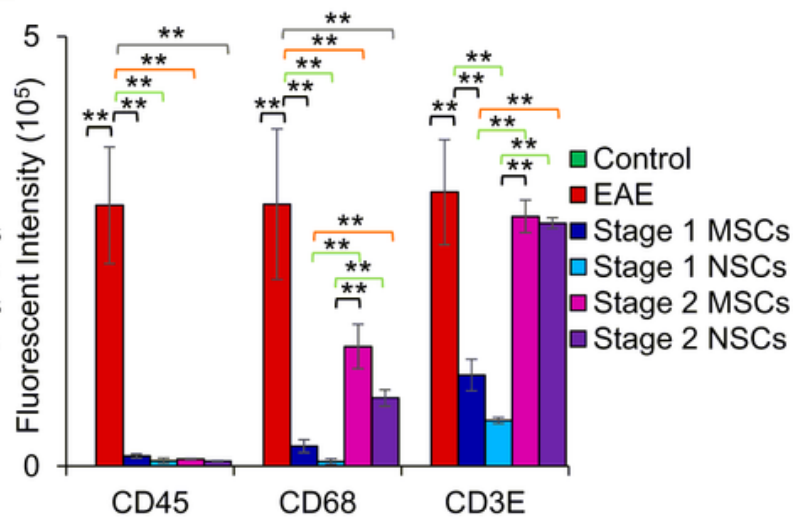

Figure 4 
Analysis of infiltrates in the CNS of EAE mice transplanted with the cells. (A) Graphical representation of Treg (CD4+CD25+) and Th17 (CD4+IL-17A+) cells, respectively, in the blood, spleen, brain, and spinal cord as determined by flow cytometry ( $* * p \leq 0.01)$. (B) Immunohistochemical staining of paraffin sections of the brain and spinal cord with CD45, CD68, and CD3E, representing leukocytes, macrophages, and T cells, respectively. ( $C$ and $D$ ) Quantification of fluorescent intensity of brain and spinal cord sections shown in $B$, respectively (**p $\leq 0.01)$.

A

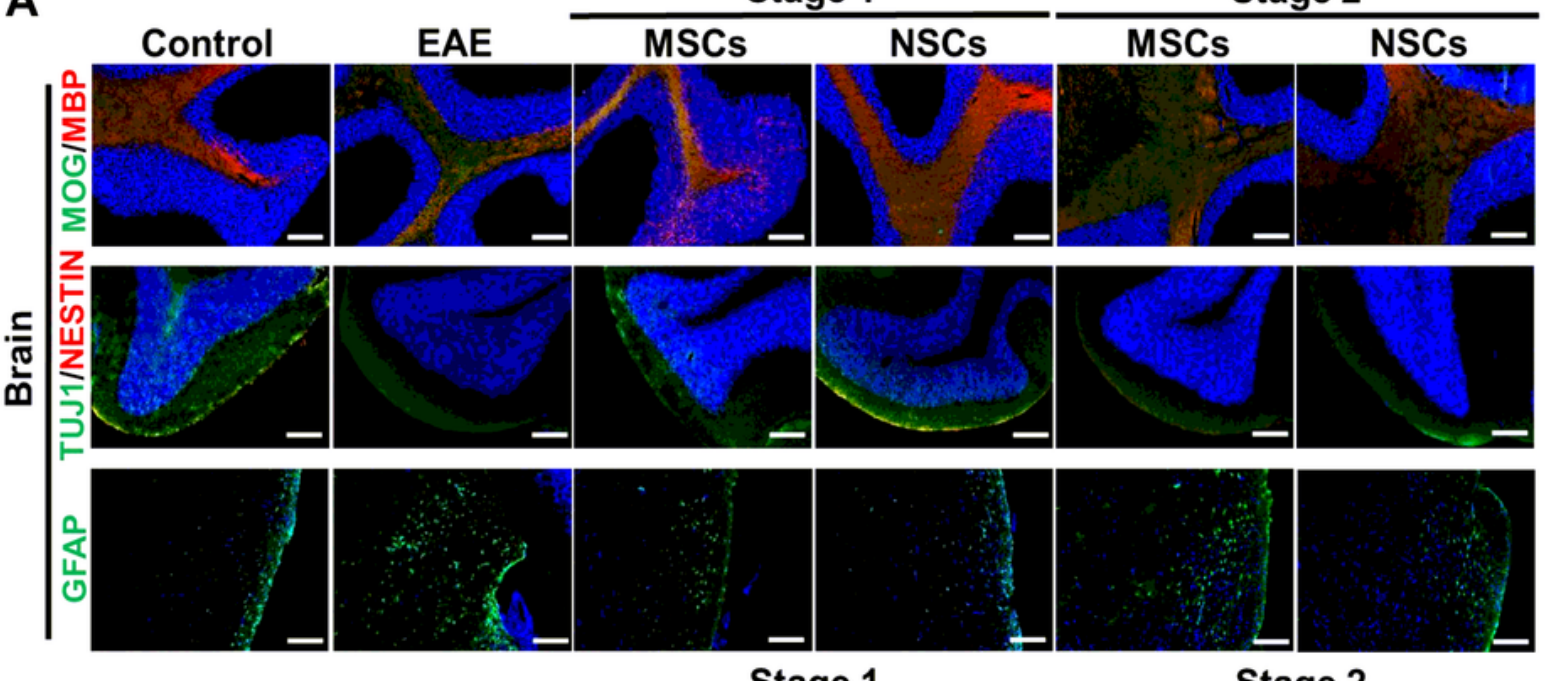

B

B Control

Stage 1

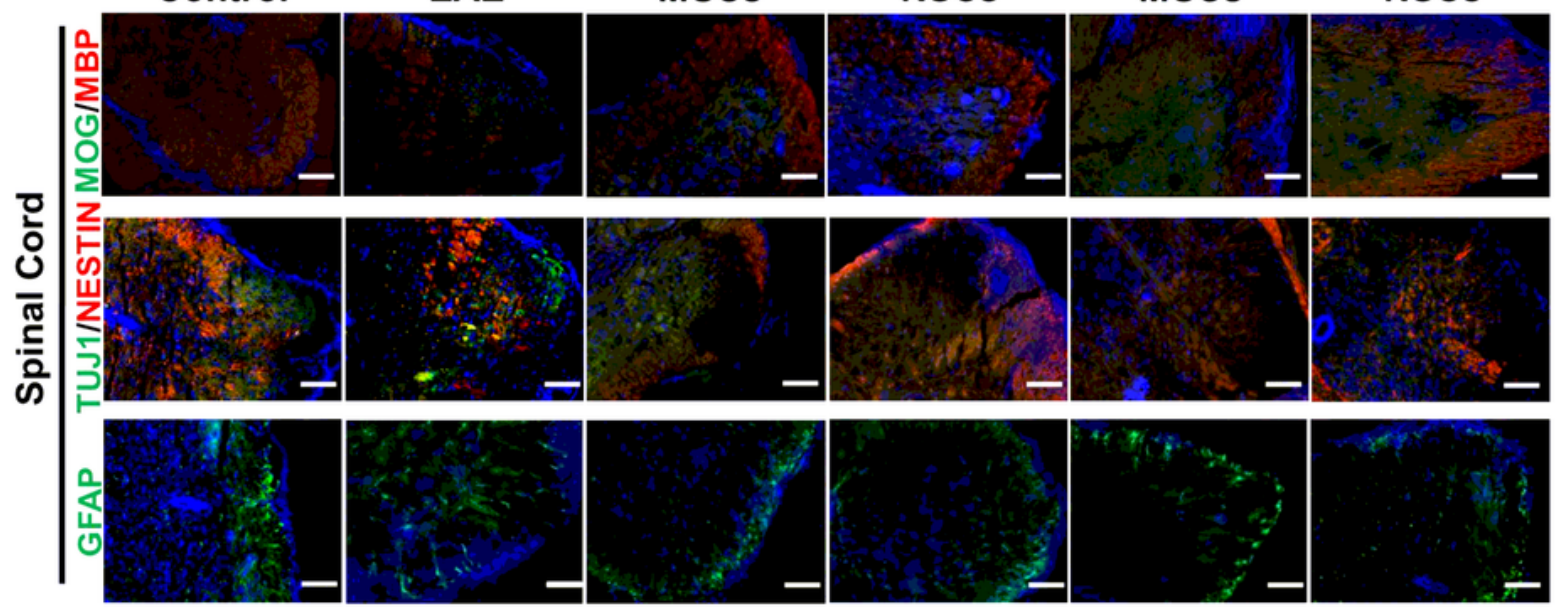

C

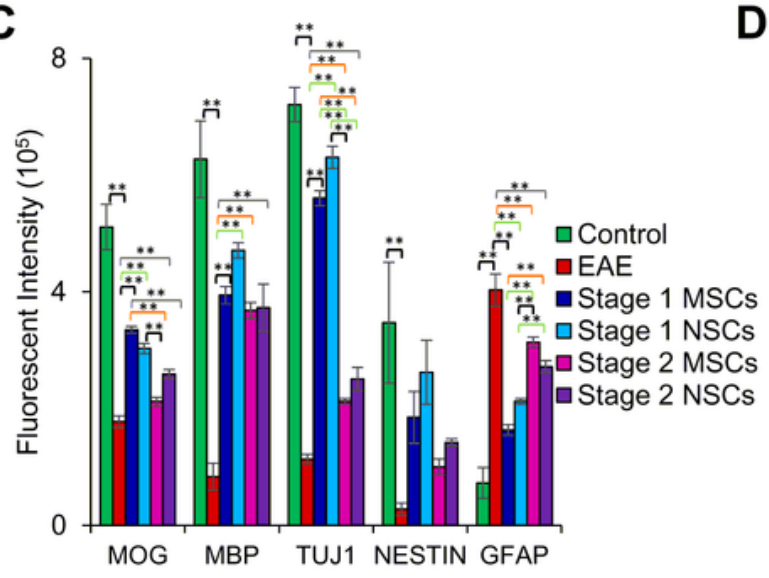

D

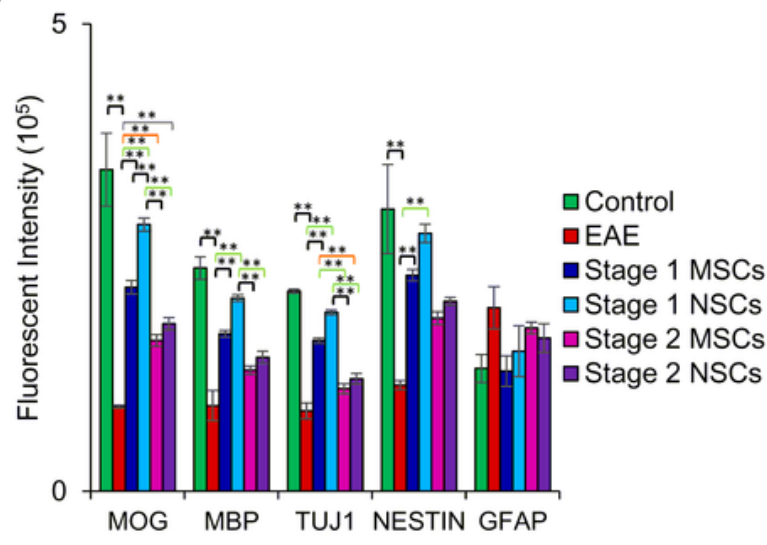

Figure 5 
Expression of neural, myelin, and astrogliosis proteins in the CNS of EAE mice transplanted with cells. (A and B) Immunohistochemical staining of paraffin sections of brain and spinal cord, respectively with neural myelin (MOG and MBP), (TUJ1 and NESTIN), and astrogliosis (GFAP) associated proteins. Scale bars represent $50 \mu \mathrm{m}$ scale bars. (Magnification: 40x). (C and D) Measurement of fluorescent intensity of proteins in the brain and spinal cord sections shown in $A$ and $B$, respectively ( ${ }^{*} p \leq 0.01$ ).

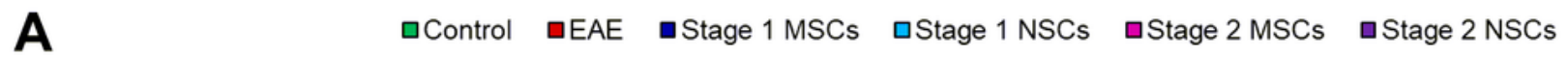

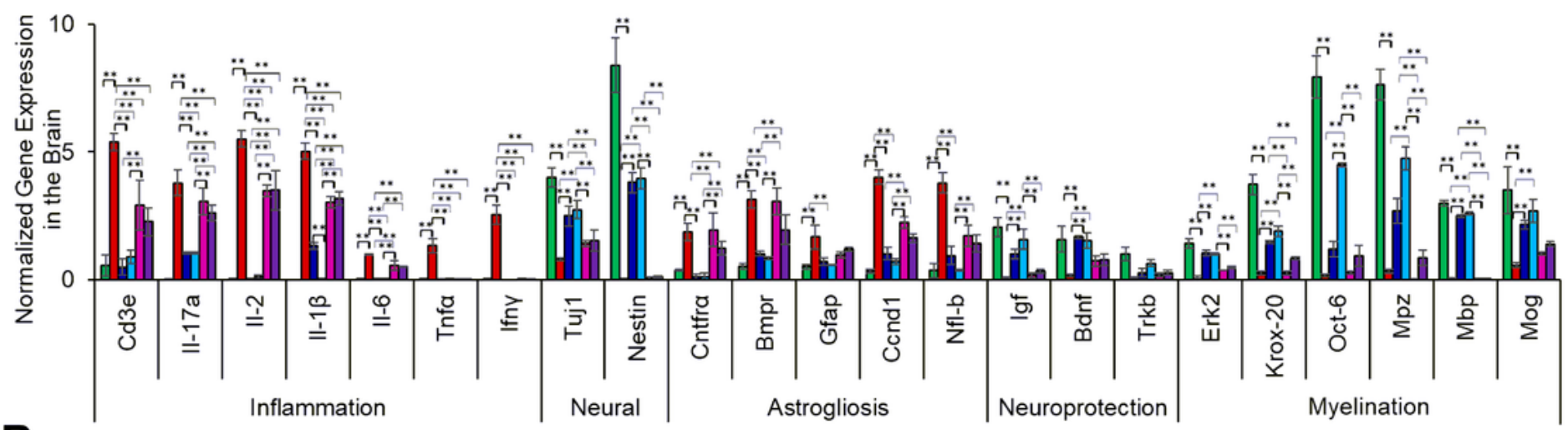

B

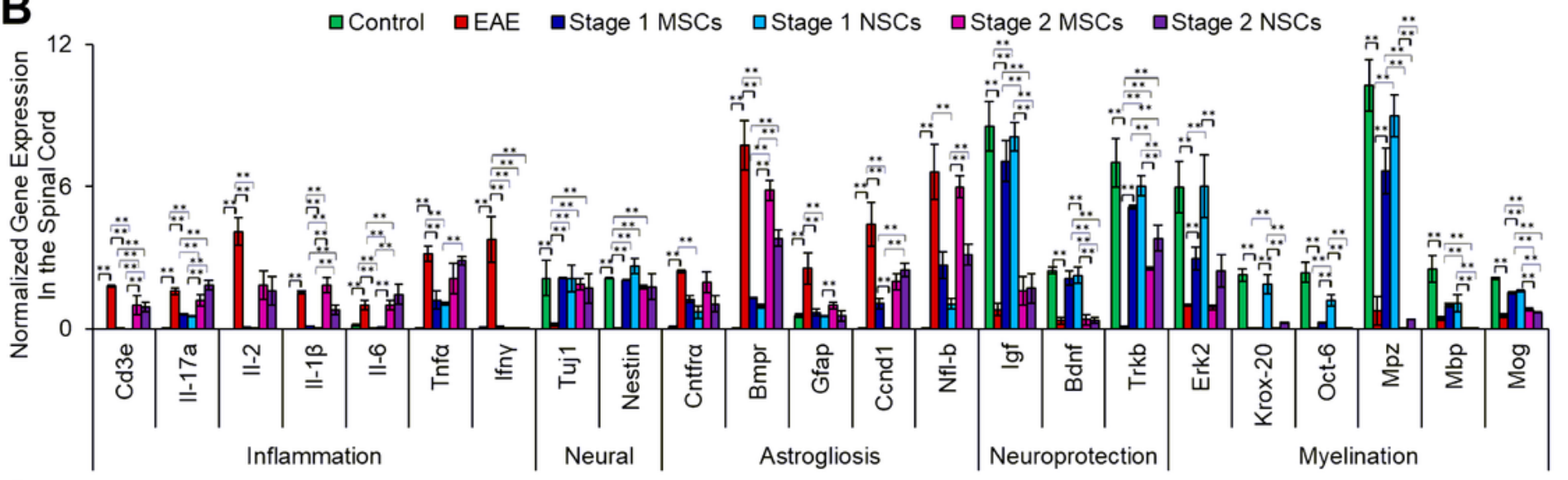

C

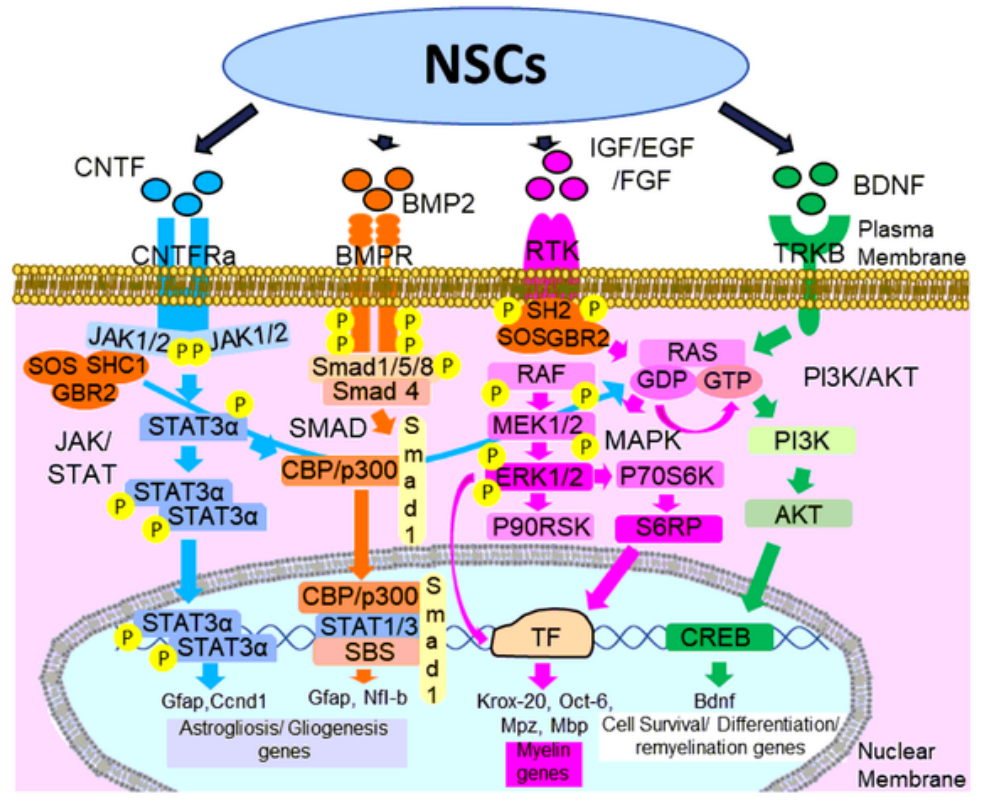

Figure 6 
Transcriptional analysis of CNS of EAE mice transplanted with cells. (A and B) Brain and spinal cord, respectively, were analyzed for the expression of endogenous inflammatory (Cd3e, II-17a, II-2, II-1 $\beta$, Tnfa and Ifny), neural (Tuj1 and Nestin), astrogliosis (Cntfr, Bmpr, Gfap, Ccnd1, and Nf1-b), neuroprotection (Igf, Bdnf, and Tkrb), and myelination (Erk2, Krox-20, Oct-6, Mpz, Mbp, and Mog) genes using qRT-PCR. Gene expression was normalized to Gapdh and Actin and error bars represent the standard error of the mean (SEM) of triplicate measures ( ${ }^{\star} p \leq 0.01$ ). (C) Proposed signaling pathways involved in neural survival, astrogliosis/gliogenesis, and myelination modulated by NSCs.

\section{Supplementary Files}

This is a list of supplementary files associated with this preprint. Click to download.

- Additionalfile1.tif

- Additionalfile2.tif

- Additionalfile3.tif

- Additionalfile4.tif

- Additionalfile5.tif 